\title{
SARS-CoV-2, multiple sclerosis, and focal deficit in a postpartum woman: A case report
}

\author{
ANCA ALEXANDRA FLOREA ${ }^{1 *}$, CARMEN ADELLA SIRBU $^{1,2}$, \\ MINERVA CLAUDIA GHINESCU ${ }^{2 *}$, CRISTINA FLORENTINA PLESA ${ }^{1,2}$, \\ ANCA MARIA SIRBU ${ }^{3 *}$, MARIAN MITRICA $^{4}$ and FLORENTINA IONITA-RADU ${ }^{2,5}$ \\ ${ }^{1}$ Department of Neurology, 'Dr Carol Davila' Central Military Emergency University Hospital, \\ Bucharest 010242; ${ }^{2}$ Department of Medical-Surgical and Prophylactic Disciplines, Faculty of Medicine, \\ Titu Maiorescu University, 031593 Bucharest; ${ }^{3}$ Department of Endocrinology, National Institute of Endocrinology, \\ CI Parhon, 011863 Bucharest; Departments of ${ }^{4}$ Neurosurgery, and ${ }^{5}$ Gastroenterology, \\ 'Dr Carol Davila' Central Military Emergency University Hospital, 010242 Bucharest, Romania
}

Received September 4, 2020; Accepted October 5, 2020

DOI: $10.3892 /$ etm.2020.9524

\begin{abstract}
SARS-CoV-2 infections raise many practical concerns in a woman with multiple sclerosis (MS) during the perinatal period. On the other hand, the impact of COVID-19 on patients with MS and disease-modifying therapies (DMTs) is unknown. We report on a female patient who was treated with interferon beta la (IFNB-1a) for many years for relapsing-remitting multiple sclerosis (RRMS) until December 2018. She developed COVID 19 infection in April 2020, after giving birth to a healthy baby girl, five weeks before. She developed a mild right hemiparesis 2 weeks later, without cold symptoms. On admission, PCR for SARS-CoV-2 was positive, and she received antivirals and corticotherapy. One month later, specific IgG and $\operatorname{IgM}$ antibodies were negative. The patient did not develop immunity to COVID-19 infection. This report raises several problems. The focal deficit could be a real relapse or a pseudo-relapse due to SARS-CoV-2 and postpartum patient vulnerability. The treatment options in this particular case raise many challenges. The absence of antibodies after a SARS-CoV-2 infection raises a big question over the acquired immunity, the increased risk of reinfection, and the subsequent evolution of MS. The standard of care for a woman with MS and COVID-19 infection during the postpartum period must be explored and more precise recommendations must be established in the future.
\end{abstract}

Correspondence to: Dr Carmen Adella Sirbu, Department of Medical-Surgical and Prophylactic Disciplines, Faculty of Medicine, Titu Maiorescu University, 67A Gheorghe Petraşcu Street, 031593 Bucharest, Romania

E-mail: sircar13@yahoo.com

*Contributed equally

Key words: multiple sclerosis, COVID-19, SARS-CoV-2, interferon beta-1a, postpartum, relapse mimic, ACE2

\section{Introduction}

SARS-COV2 is a new coronavirus that causes a respiratory infection that can manifest through a specter of symptoms, from asymptomatic to severe acute respiratory syndrome. It is now known that the disease is more severe in patients with other comorbidities, but it remains unclear how the infection affects people with autoimmune diseases and particularly patients with multiple sclerosis (MS). MS is a disease characterized by relapses and a link between infections and MS exacerbation is suspected. There is evidence that suggests that relapses associated with infections increase the risk of sustained neurological deficit compared to relapses not associated with infections (1). Although newer disease-modifying treatments (DMTs) can increase the risk of infections, interferon $\beta$, which is an older DMT, is not associated with infections and even has antiviral proprieties $(2,3)$. A lot of research is still to be done around the world regarding COVID-19, but at the base of all the research, remain clinical observation and case reports.

\section{Case report}

Multiple sclerosis and COVID-19. We report a case of SARS-CoV-2 infection in a 40-year-old Caucasian woman with recurrent remissive multiple sclerosis (RRMS), which began 5 weeks postpartum and was associated with a focal neurological deficit. Her medical history was insignificant. The first brain and cervical MRI examination in 2012, revealed numerous demyelinating lesions, some of which were active. She underwent chronic treatment with subcutaneous interferon beta 1a (IFNB-1a) 3 times per week, in October 2012. MRI and clinical evaluation one year later showed no disease activity. First, she stopped the treatment in May 2014, for 16 months, for a possible pregnancy. Brain and cervical MRI performed at the therapy resumption showed new lesions. In August 2018, she developed a mild right hemiparesis, remitted under corticotherapy, and in December 2018 she stopped again the treatment for pregnancy. 
On the 10th of February 2020, a healthy baby girl was born and breastfeeding began. Five weeks postpartum, the patient attended for paresthesia and disability in the right limbs with the onset two weeks before, with stable evolution. She had exited twice from isolation (on the 10th of March for a neonatological, and on the 16th of March for a gynecological consultation, in the outpatient department).

At admission, hemoglobin and hematocrit were increased, [HGB $16.5 \mathrm{~g} / \mathrm{dl}$ (11.10-14.70), HCT 50.1\% (35-47)]. The D-dimers had a value of $67 \mathrm{ng} / \mathrm{ml}(0-250)$, she had normal leukocytes with mild lymphopenia and granulocytosis, which worsened during hospitalization, C-reactive protein (CRP) of $7,15 \mathrm{mg} / \mathrm{l}(0.00-5.00)$, and positive testing with polymerase-chain-reaction (PCR) for SARS-CoV-2. On the chest $\mathrm{X}$-rays, a basal infiltration was described. She presented a motor deficit and mild ataxia in the right limbs (4/5 MRC), brisk reflexes, good general condition, without cold symptoms. According to the hospital protocol, she was transferred to the Infectious Diseases Hospital designated for the treatment of positive patients.

It was considered an MS relapse and the patient was given methylprednisolone $1 \mathrm{~g}$ /day for 3 days, with persistent symptoms. She started specific therapy (hydroxychloroquine $4 \mathrm{~g} /$ day, lopinavir/ritonavir 4 tablets/day for 10 days, and azithromycin $1 \mathrm{~g} / \mathrm{day}$, for 3 days). Three days later, she went to the Neurology Department for positive patients where she was kept in isolation for up to 14 days. She was discharged after two consecutive, negative PCRs. A slight right brachial motor deficit is maintained (pronator drift). After 2 weeks, PCR, $\mathrm{IgG}$, and IgM for SARS-CoV-2 were negative, along with the remission of the neurological deficit. Instead, D-dimer values showed an increasing tendency.

\section{Discussion}

On the 30th of April, 2020, in Romania, there were 12,240 COVID 19 cases registered, with a maximum incidence of $24 \%$ for the age group of 40-49 (predominantly females), with a mortality rate of $6 \%$. It is possible that at one of the two postpartum medical examinations, the patient may have contracted the virus (given its average incubation period and intra-community transmission). The severity of the disease and mortality was higher in women in the perinatal period, as shown by the previous pandemics with SARS and H1N1 (4). Although there is no current evidence of vertical or breast transmission of the virus, there is a possibility of newborn infection by droplets from the mother. In our particular case, the infant was kept separate from his mother and was not breastfed. Breast milk is a natural source of antibodies, and mothers are encouraged to pump while being isolated from the baby. In this case, however, given the antiviral medication, breastfeeding was permanently discontinued. The child was not tested, but was permanently asymptomatic, with good development.

We do not know whether the risk of COVID-19 infection is higher in MS patients. We also do not know whether mild or moderate, self-limiting respiratory infections, which did not require medical attention, have a higher share among MS patients. In patients with MS and mild viral comorbidities, including COVID-19 infection, immunomodulatory treatment with DMT is continued (5). Our patient discontinued treatment in December 2018, for a pregnancy. Continuation of IFNB-1a may have protected her from SARS-CoV-2, given its antiviral properties (6). For severe COVID 19 infections, DMT was discontinued and resumed after 4 weeks, or when symptoms resolve completely.

The use of corticosteroid therapy, in this case, is debatable. The neurological deficit was mild and corticosteroid therapy could worsen the course of the infection. High doses of steroids accelerate the remission of the relapses but do not influence the final degree of recovery. Therefore, a better risk/benefit assessment is recommended for patients with MS and COVID-19 infection.

The patient's neurological deficit may have been a worsening of older symptoms in the viral context (relapse mimic). For this scenario we have 3 explanations: She had the same type of focal deficit in 2018 before stopping IFNB-1a; remission of her deficit was delayed, to the end of antiviral therapy, not after the methylprednisolone cure; the absence of active lesion on MRI performed 6 weeks after the onset of the current deficit.

Thus, it may have been a pseudo relapse and the opportunity to administer corticosteroids in a confirmed viral context should be well appreciated. Considering the possibility of a relapse mimic, it was preferable to delay the methylprednisolone therapy until obtaining the PCR result (approximatively $24 \mathrm{~h}$ ) and perform an MRI examination. On the other hand, a recommendation during pandemics is the use of oral corticosteroids in equivalent doses to injectables, to allow patients to be isolated at home and to limit the risk of contamination.

New neurological disorders are reported in the context of COVID-19 infection, given the neurotropism and neuroinvasiveness of the nervous system (7). We could speculate in this case the impaired microcirculation and prothrombotic states, by SARS-CoV-2 action on angiotensin-converting enzyme 2 (ACE2) functional receptor in endothelial cells, as well as cytokines release. The hemoconcentration and the postthrombotic state during postpartum could also have contributed to this focal deficit. The rising values of D-dimers up to one month after onset may be an argument for this assumption. Evaluation of the prothrombotic profile of the patient would be necessary. The absence of antibodies after a SARS-CoV-2 infection raises a big question over the acquired immunity, the increased risk of reinfection, and the subsequent evolution of MS. We need a new quality standard of care for women who give birth and MS in pandemic time (8). These aspects are important to resume DMTs as soon as possible, in postpartum. Immunomodulatory treatment such as Interferons have no increased risk of systemic infections, so resuming the IFNB-1a may be a correct option in this case $(9,10)$. While waiting for a vaccine, the completion of which has not materialized for SARS or MERS, so far, a balanced lifestyle, with physical therapy, melotherapy, healthy eating, can lead to a natural increase in immunity (11-14). Also, new monoclonal antibody therapies that reduce lymphocytes must be weighed well before administration during the pandemic (15).

The neurological deficits that appear in MS patients must be very carefully analyzed. They are predictable, part 
of the natural course of the disease, but can sometimes be pseudo-relapses. In these situations, corticosteroid therapy may be well balanced. Pathogenic mechanisms can interfere with MS, DMTs, and viral infections. Women with MS in the postpartum period are even more vulnerable to relapses and infections, and the management of these cases is a state of the art, and need a new standard of care.

\section{Acknowledgments}

Not applicable.

\section{Funding}

No funding was received.

\section{Availability of data and materials}

Not applicable.

\section{Authors' contributions}

AAF, CAS, MCG, MM contributed in the design and conception of the study. FIR, CAS, CFP, AMS, MM and MCG were involved in selection, analysis, and interpretation of cited references. Moreover, all the authors were involved in the drafting and reviewing of this paper as well as revising it critically for important intellectual content. All authors read and approved the final version of the manuscript and agreed to be accountable for all aspects of the study.

\section{Ethics approval and consent to participate}

The study was approved by the Ethics Committee of 'Dr Carol Davila' Central Military Emergency University Hospital (Bucharest, Romania). Written informed consent was obtained from the patient.

\section{Patient consent for publication}

Written informed consent was obtained from the patient.

\section{Competing interests}

The authors declare that they have no competing interests.

\section{References}

1. Libbey JE, Cusick MF and Fujinami RS: Role of pathogens in multiple sclerosis. Int Rev Immunol 33: 266-283, 2014.

2. Coles AJ, Twyman CL, Arnold DL, Cohen JA, Confavreux C, Fox EJ, Hartung HP, Havrdova E, Selmaj KW, Weiner HL, et al: Alemtuzumab for patients with relapsing multiple sclerosis after disease-modifying therapy: A randomised controlled phase 3 trial. Lancet 380: 1829-1839, 2012.

3. Shirani A, Zhao Y, Karim ME, Evans C, Kingwell E, van der Kop ML, Oger J, Gustafson P, Petkau J and Tremlett H: Association between use of interferon beta and progression of disability in patients with relapsing-remitting multiple sclerosis. JAMA 308: 247-256, 2012.

4. Breslin N, Baptiste C, Gyamfi-Bannerman C, Miller R, Martinez R, Bernstein K, Ring L, Landau R, Purisch S, Friedman AM, et al: Coronavirus disease 2019 infection among asymptomatic and symptomatic pregnant women: Two weeks of confirmed presentations to an affiliated pair of New York City hospitals. Am J Obstet Gynecol MFM 2: 100118, 2020.

5. Giovannoni G, Hawkes C, Lechner-Scott J, Levy M, Waubant E and Gold J: The COVID-19 pandemic and the use of MS disease-modifying therapies. Mult Scler Relat Disord 39: 102073, 2020.

6. Sîrbu CA, Drăgo CM, Nicolae AC and Pleșa CF: History of interferon treatments in multiple sclerosis - 60 years of progress. Farmacia 65: 14-18, 2017.

7. Wu Y, Xu X, Chen Z, Duan J, Hashimoto K, Yang L, Liu C and Yang C: Nervous system involvement after infection with COVID-19 and other coronaviruses. Brain Behav Immun 87: 18-22, 2020.

8. Hobart J, Bowen A, Pepper G, Crofts H, Eberhard L, Berger T, Boyko A, Boz C, Butzkueven H, Celius EG, et al: International consensus on quality standards for brain health-focused care in multiple sclerosis. Mult Scler 25: 1809-1818, 2019.

9. Brownlee W, Bourdette D, Broadley S, Killestein J and Ciccarelli O: Treating multiple sclerosis and neuromyelitis optica spectrum disorder during the COVID-19 pandemic. Neurology 94: 949-952, 2020.

10. Sirbu CA,Furdu-LungutE, Plesa CF, Nicolae AC and Drăgoi CM: Pharmacological treatment of relapsing remitting multiple sclerosis - where we are throw evidence based medicine? - review. Farmacia 64: 651-656, 2016.

11. Docea AO, Tsatsakis A, Albulescu D, Cristea O, Zlatian O, Vinceti M, Moschos SA, Tsoukalas D, Goumenou M, Drakoulis N, et al: A new threat from an old enemy: Re-emergence of coronavirus (Review). Int J Mol Med 45: 1631-1643, 2020.

12. Calina D, Docea AO, Petrakis D, Egorov AM, Ishmukhametov AA, Gabibov AG, Shtilman MI, Kostoff R, Carvalho F, Vinceti M, et al: Towards effective COVID-19 vaccines: Updates, perspectives and challenges (Review). Int J Mol Med 46: 3-16, 2020.

13. Sirbu CA, Sirbu OM, Constantin C and Sandu A: Neuroimunotoxicity of aluminum. Farmacia 63: 8-10, 2015.

14. Stroe ZA,Docu-Axelerad S and Docu-Axelerad D: Melotherapy - A complementary approach in the symptomatology of multiple sclerosis patients. J Complement Med Res 11: 164-169, 2020.

15. Sirbu CA, Budisteanu M and Falup-Pecurariu C: Monoclonal antibodies - a revolutionary therapy in multiple sclerosis. Neurol Neurochir Pol 54: 21-27, 2020. 